\title{
Performance Analysis of Magnetic Power Pads for Inductive Power Transfer Systems with Ferrite Structure Variation
}

\author{
Minkook Kim*, Jongeun Byun* and Byoung Kuk Lee ${ }^{\dagger}$
}

\begin{abstract}
In this paper, performance of rectangular shaped magnetic power pads for inductive power transfer (IPT) system according to ferrite structure is analyzed. In order to evaluate the influences of ferrite structure, six cases of magnetic power pads are proposed. Self-inductance, coupling coefficient, quality factor, and coil to coil efficiency are compared as the displacement increases in the direction of $\mathrm{x}$ or $\mathrm{y}$ axis. For accurate estimation, finite element method (FEM) simulation is used and loss components of the power pads are numerically calculated and considered. Through the simulation and measured results, effectiveness of protrusive and enveloping ferrite structure is identified.
\end{abstract}

Keywords: Coupling coefficient, Ferrite structure, Finite element method, Inductive power transfer, Wireless power transfer

\section{Introduction}

As the interests on the eco-friendly vehicles increase, researches on inductive power transfer (IPT) chargers for electric vehicles are active. As shown in Fig. 1, the IPT system is composed of several power converters and magnetic power pads. In comparison with the efficiency of the power converter stages, the one of the magnetic power pads is fairly low. Thus, in the IPT system, magnetic power pads significantly affect the system performance. Because IPT system is a loosely coupled system, magnitude of coupling between primary power pad and secondary power pad is one of the main figure of merits (FOM) [1, 11]. The coupling of the magnetic power pads in the EVs is influenced by vertical gap and horizontal misalignment. For maintaining system performance over operational range, coupling coefficient of the magnetic power pads should be constant regardless of horizontal misalignment. In order to satisfy these trends, SAE J2954, which is a standard for wireless charging of electric and plug-in hybrid vehicles, is legislated and the rectangular shape of primary power pads is decided [2]. In general, basic component of the magnetic power pads is Litz wire for stable and reliable operation at high frequency. However, solely used Litz wire cannot reduce the leakage flux of the magnetic power pads. Therefore, on the purpose of improving the coupling and reducing leakage flux, ferrite and aluminum shield are used practically. By adding ferrite in the magnetic power pads, both inductance and coupling are increased and this tendency is directly influenced by structure of ferrite [3]. Previous studies only analyzed the effect of coils in

$\dagger$ Corresponding Author: Department of Electrical and Computer Engineering, Sungkyunkwan University, Korea. (bkleeskku@skku.edu)

* Department of Electrical and Computer Engineering, Sungkyunkwan University, Korea (mkfour44@skku.edu)

Received: December 28, 2016; Accepted: February 27, 2017 magnetic power pads numerically, so it is limited to predict the performance alternation by ferrite structure [4]. Since the numerical analysis considering effects of both coil and ferrite is not easy, finite element method (FEM) simulation is used to estimate the magnetic characteristics according to the ferrite structure variation [5].

In this paper, the six cases of ferrite structure in rectangular shaped magnetic power pads is proposed and the performance is analyzed in detail. For accurate analysis, coil losses, considering skin effect and proximity effect, is calculated. The losses of the ferrite and aluminum are obtained through FEM simulation. Taking into account of loss components, self-inductance, coupling coefficient $k$, quality factor $Q$, and coil to coil efficiency $\eta$ are analyzed and magnetic flux density of six cases are compared under both vertical gap and horizontal misalignment. Based on the simulation results, two cases of the ferrite structure, which are flat plate ferrite and flat plate ferrite with prolonged ferrite at outermost, are manufactured and their magnetic performance are compared.

\section{Parameters of Magnetic Power Pads}

The transmission efficiency of the magnetic power pads is determined by coupling coefficient and quality factors of primary and secondary magnetic power pads. These parameters can be calculated by deriving self/mutual inductances and equivalent resistances of the magnetic power pads [6]. Self-inductance of the primary pad is easily obtained by applying current to the primary coil while secondary coil is open-circuited and self-inductance of the secondary pad can be acquired in an opposite way. The coupling coefficient is calculated by using the conventional ac circuit definition of $k$ as (1). 


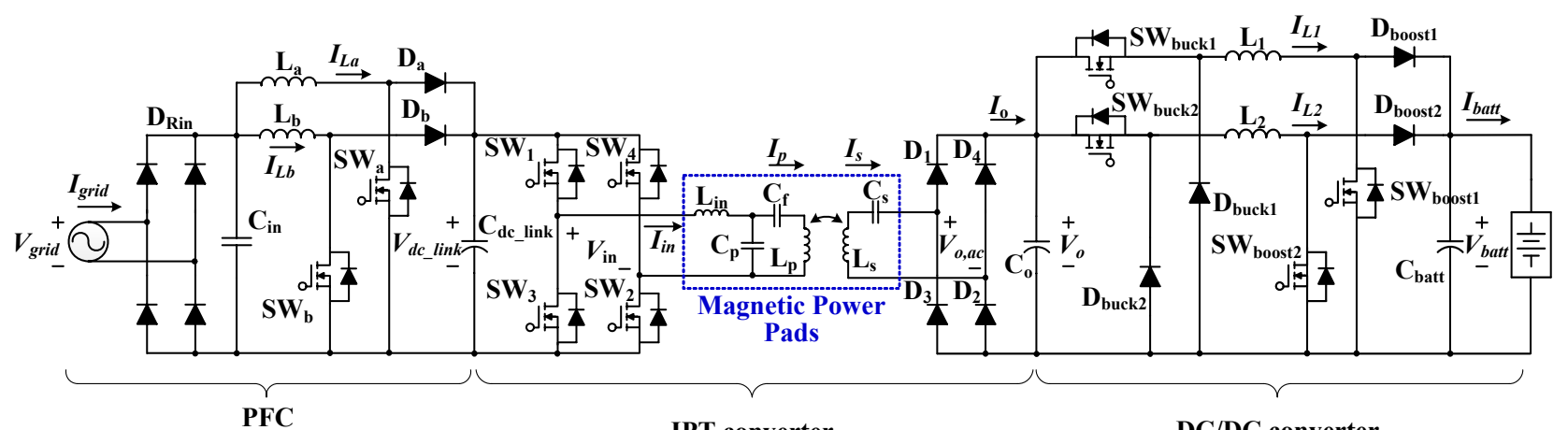

converter

IPT converter

DC/DC converter

Fig. 1. Schematic of the IPT system

$$
k=\frac{M}{\sqrt{L_{p} L_{s}}}
$$

where, $M$ is mutual inductance, $L_{p}$ is primary selfinductance, and $L_{s}$ is secondary self-inductance.

Quality factor is a ratio of the energy stored in the oscillating resonator to the energy dissipated, which can be calculated using self-inductance, operating frequency, and loss component of the magnetic power pads.

$$
Q_{p, s}=\frac{\omega L_{p, s}}{R_{p, s}}
$$

where, $\omega$ is operating angular frequency, and $R_{p}$ and $R_{s}$ are equivalent resistances for primary and secondary power pads derived by magnetic power pad losses. For high frequency applications, Litz wire is used to reduce both skin effect and proximity effect. The equivalent resistance of magnetic power pad consists of $R_{\text {coil }}$ and $\Delta R$.

$$
R=R_{\text {coil }}+\Delta R
$$

where, $R_{\text {coil }}$ is coil resistance, categorized into $R_{\text {skin }}$ by skin effect and $R_{\text {prox }}$ by proximity effect. In case of $R_{\text {skin }}$, and it also includes the dc resistance [7]. Eqs. (4)-(7) show the formula for calculating total length of the rectangular power pad, skin effect loss, proximity loss for single turn, and total proximity effect loss, respectively.

$$
\begin{gathered}
l_{\text {rect }}=\sum_{n=1}^{N}\left[4\left(a_{1}+(n-1) b_{1}\right)+b_{1}+4\left(a_{2}+(n-1) b_{2}\right)\right] \\
P_{\text {skin }}=n \cdot \frac{4 \cdot l_{\text {rect }}}{n \sigma \pi d_{i}^{2}} \cdot F_{R}(f) \cdot\left(\frac{\hat{I}}{n}\right)^{2} \\
P_{\text {prox }}=n \cdot \frac{4 \cdot l_{\text {rect }}}{n \sigma \pi d_{i}^{2}} \cdot G_{R}(f) \cdot\left(\hat{H}_{e}^{2}+\frac{I^{2}}{2 \pi^{2} d_{a}^{2}}\right) \\
P_{\text {prox_total }}=\sum_{i=1}^{N} P_{\text {prox }(i)} \cdot l_{\text {rect }}
\end{gathered}
$$

where, $n$ is the number of strand, $a_{1,2}$ are two side lengths

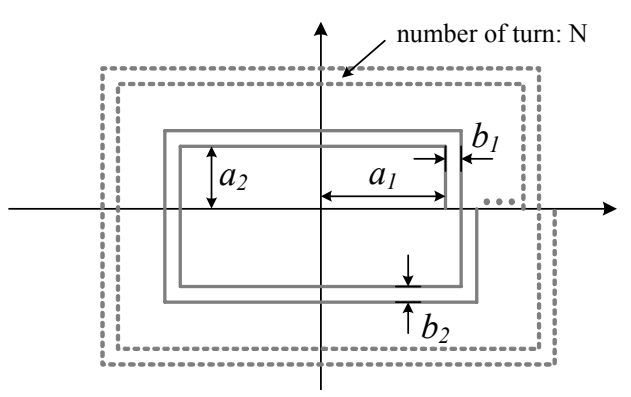

Fig. 2. Expression of the rectangular shaped power pad

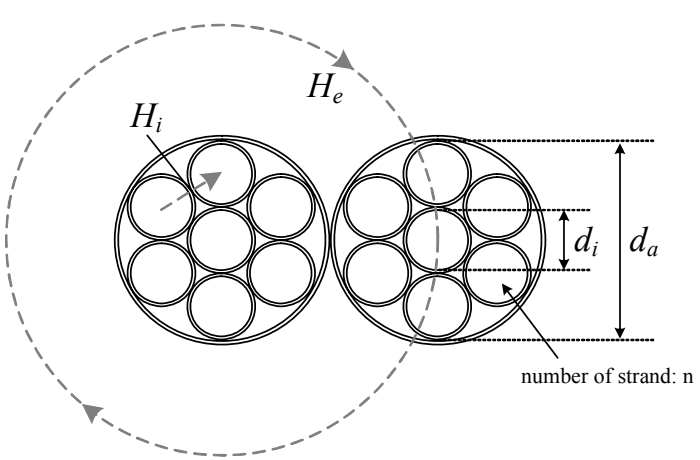

Fig. 3. Cross-section of Litz wire windings

of the rectangular coils, $b_{1,2}$ are distance between each turn for horizontal and vertical direction, $N$ is the total number of turn for rectangular winding, $\sigma$ is conductivity of copper, $d_{i}$ is diameter of one strand, $F_{R}$ is a resistance increase ratio caused by skin effect, $G_{R}$ is a resistance increase ratio caused by proximity effect, and $H_{e}$ is external magnetic field strength. Utilizing (5) and (7), equivalent resistance of Litz wire, which reflects the skin effect and proximity effect, is derived as

$$
R_{\text {coil }}=R_{\text {skin }}+R_{\text {prox }}=\frac{P_{\text {skin }}}{I_{r m s}{ }^{2}}+\frac{P_{\text {prox_total }}}{I_{r m s}{ }^{2}}
$$

where, $I_{r m s}$ is rms value of applied ac current to the coil. Figs. 2 and 3 illustrate the windings of the rectangular power pad and cross-section of Litz wire winding to 
explain the proximity effect.

$\Delta R$ is resistance component by the ferrite and aluminum shield seen from either primary or secondary side. In order to induce $\Delta R$, firstly, losses of the primary and secondary side ferrite and aluminum should be calculated when ac current is applied to the coil while opposite side coil is open-circuited. Secondly, dividing the total losses of ferrite and aluminum by square of the applied ac current.

$$
\Delta R=\frac{\sum\left(P_{F e, p r i}+P_{A l, p r i}+P_{F e, \mathrm{sec}}+P_{A l, \mathrm{sec}}\right)}{I_{r m s}^{2}}
$$

where, $P_{F e, p r i s e c}$ are ferrite losses of primary and secondary side, and $P_{A l, p r i s e c}$ are aluminum shield losses of primary and secondary side. Total power losses of the magnetic power pads can be calculated thereby modeling the magnetic power pads with designed electrical parameters and substituting the derived resistance by (3) to the coil resistance in the FEM simulation.

In order to evaluate the efficiency at the desired power, which is $3.3 \mathrm{~kW}$ in this paper, load resistance is determined practically using circuital analysis simulation based on output power and open circuit voltage $\left(\mathrm{V}_{\mathrm{oc}}\right)$ when ac current is applied to the primary coil. The described parameters are used as index of evaluation in the following section.

\section{Magnetic Parameter Comparison According to the Ferrite Structure}

General structure of ferrite in the previous studies on rectangular power pads is either flat plate ferrite for whole pad or bar ferrite aligned in the vertical or horizontal direction [8]. With regard to the bar ferrite, the flux leakage between ferrite bars degrades the performance and increases eddy current losses. However, by adopting the flat plate ferrite, above problems can be mitigated. Thus, intensive research on ferrite structure for enhancing the performance of magnetic power pad is necessary. Therefore, in this paper, so as to compare the performance in accordance with the ferrite structure, six magnetic power pads with different ferrite structure are proposed. Fig. 4 shows the configuration and dimension of the magnetic power pad with flat plate ferrite structure (case 1). The units for the values in the parentheses are mm. Except for ferrite structure, configuration and dimensions for other five cases are identical. Fig. 5 illustrates the modeling of six cases of magnetic power pads with the following structures.

Case 1: basic structure, flat plate ferrite

Case 2: protrusive ferrite at center of the power pad with vacant space

Case 3: protrusive ferrite at center and prolonged ferrite

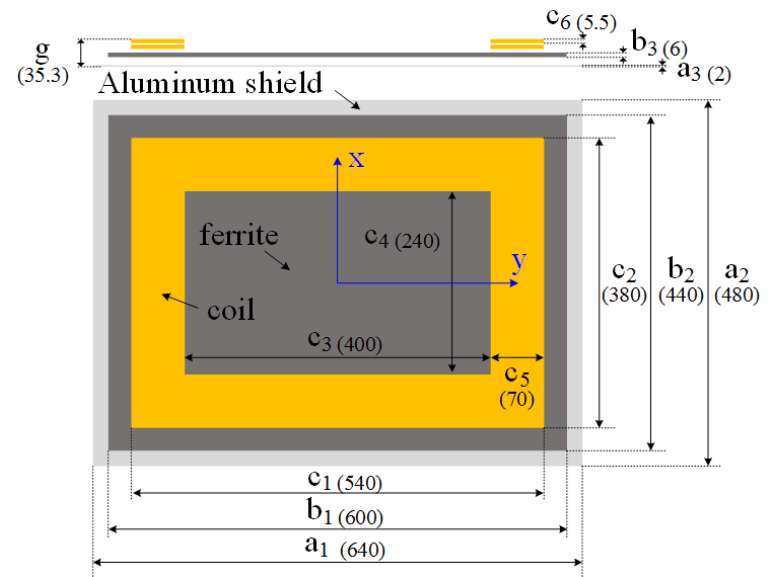

(a) primary side

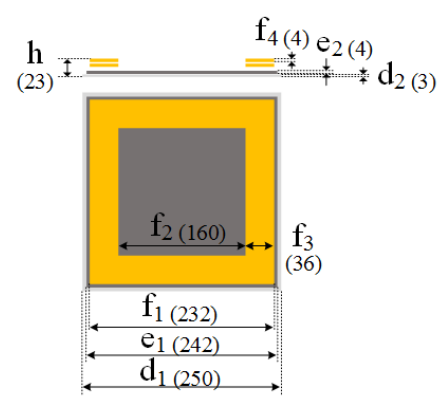

(b) secondary side

Fig. 4. Configuration and dimension of the power pads.

at outermost

Case 4: flat plate ferrite with prolonged ferrite at outermost

Case 5: flat plate ferrite with empty space at center

Case 6: enveloping coil windings with ferrite

All modellings in Fig. 5 are half model to depict the cross section of the ferrite structure. Regardless of the ferrite structure, thickness of ferrite for each case is identical as $6 \mathrm{~mm}$. In order to evaluate the effects of primary side ferrite structure, simulations are performed to compare self-inductance of primary and secondary pad $L_{p}$, $L_{s}$, coupling coefficient $k$, quality factors of primary and secondary pad $Q_{p}, Q_{s}$, and coil to coil efficiency between primary and secondary power pad in accordance with $\mathrm{x}$ or $\mathrm{y}$ axis horizontal misalignment $(0 \sim 120 \mathrm{~mm}$ ( $\mathrm{x}$ axis), 0 200 mm (y axis), $40 \mathrm{~mm}$ step) at $100 \mathrm{~mm}$ vertical gap. The maximum misalignment distance for each axis are determined by $120 \mathrm{~mm}$ and $200 \mathrm{~mm}$ since the performance of the power pad is drastically decreased when the inner side of the secondary coil is deviated from that of the primary coil. Operating and resonant frequency are set by $85 \mathrm{kHz}$ and applied current to the primary coil is $16.83 \mathrm{~A}_{\mathrm{rms}}$ The coils are double-layered and the number of coil turns for primary and secondary power pads are set by 20 turns, respectively. 


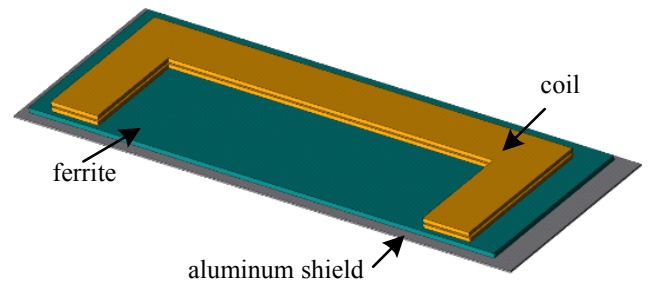

(a) case 1

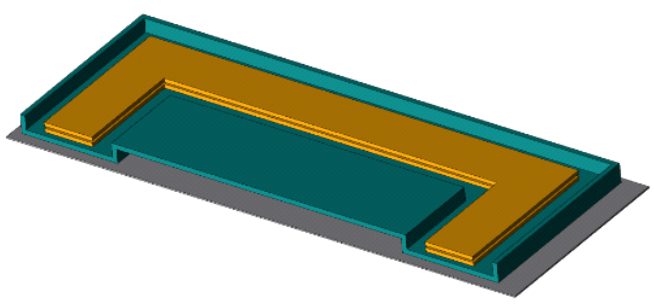

(c) case 3

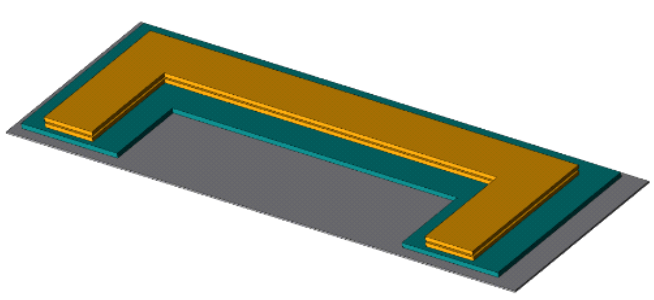

(e) case 5

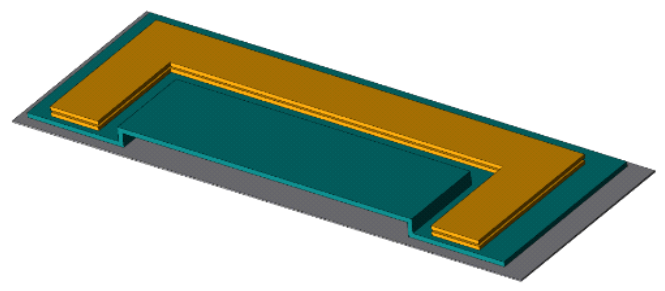

(b) case 2

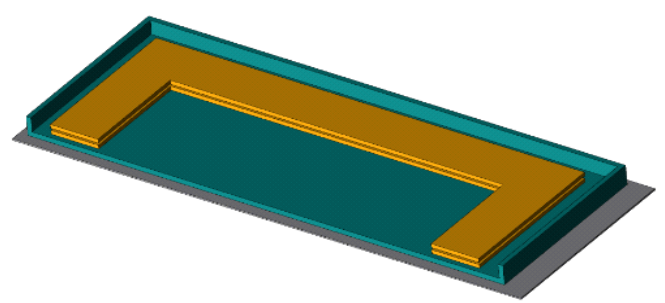

(d) case 4

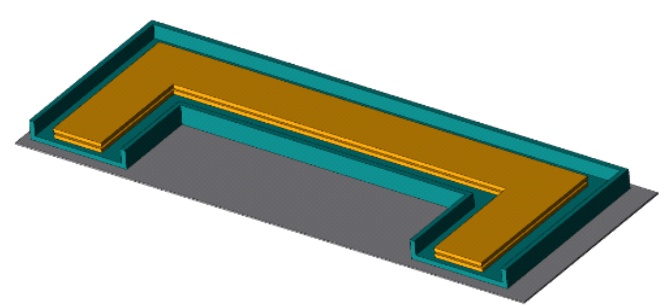

(f) case 6

Fig. 5. Proposed magnetic power pad modellings with different primary side ferrite structures

\subsection{Self-inductance}

In general, switching frequency of the IPT system is designed for either zero phase angle (ZPA) or zero voltage switching (ZVS) operation to minimize VA rating and power losses. However, resonant frequency of the IPT system varies as the displacement increases since both selfinductance of the primary and secondary power pads are changed. Thus, change in self-inductance according to the misalignment is an important parameter. Figs. 6(a) and (b) show the $L_{p}$ when the secondary pad is horizontally misaligned. As can be seen, $L_{p}$ of cases 2, 3, 4, and 6 are larger and case 5 is smaller than that of the case 1 . The $L_{p}$ increases regardless of ferrite structure as horizontal displacement distance increases. This is because, when the secondary pad is smaller than the primary pad, aluminum shield on the secondary pad lowers the primary pad inductance as the primary and secondary pads become closer [9]. Secondary pad inductances $L_{s}$ are also simulated and show almost constant value regardless of misalignment because the ferrite of the primary pad is relatively larger than the size of secondary power pad. Figs. 6(c) and (d) show the $L_{s}$ when the secondary pad is horizontally misaligned.

\subsection{Coupling coefficient}

In order to calculate coupling coefficient of the magnetic power pads using (1), $L_{p}, L_{s}$ and mutual inductance $M$, are derived. Figs. 6(e) and (f) depict the $k$ as the secondary power pad is misaligned. Cases 2 and 3 show higher $k$ than case 1 because protrusive ferrite at center lowers the pad reluctance and helps flux flow. The $k$ of case 4 is similar to that of case 1 and case 5 shows the worst performance. Since the center part of the primary side ferrite is empty, the coupling decreases as the misalignment increases until inner side of the secondary coil is deviated from the ferrite empty area. Case 6 shows the worse coupling than case 1 at the initial point, however, as the displacement increases, the magnitude of coupling is similar to that of the case 1 .

\subsection{Quality factor}

As mentioned at self-inductance part, except case 5, other cases represent larger $L_{p}$ than case 1 . In case of the equivalent primary pad resistance $R_{p}$, cases 2 and 3 are larger, cases 5 and 6 are smaller, and case 4 is similar to that of the case 1 . Using (2), quality factors of six cases are derived. Figs. $6(\mathrm{~g})$ and $(\mathrm{h})$ illustrate the $Q_{p}$ of the six cases. The $Q_{p}$ of 6 cases increase as the misalignment distance increases. This is because the eddy current losses of the secondary pad decreases as the displacement increases and this results in reduction of $R_{p}$. Secondary pad quality factor $Q_{s}$, is also simulated and does not vary substantially because of constant secondary pad inductance and equivalent secondary resistance $R_{s}$. Figs. 6(i) and (j) 


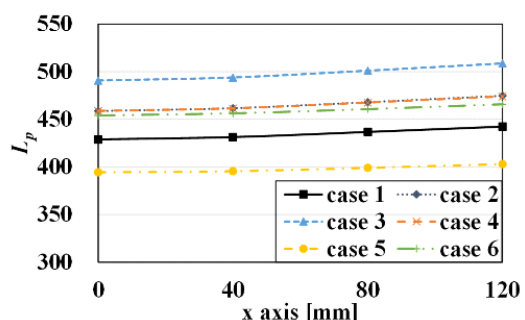

(a)

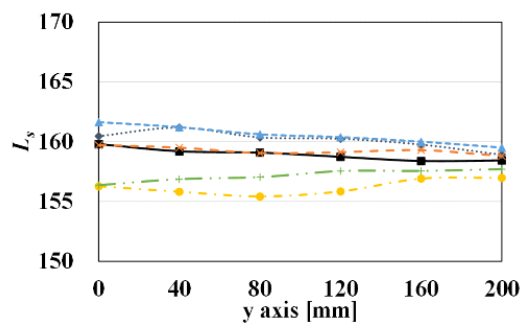

(d)

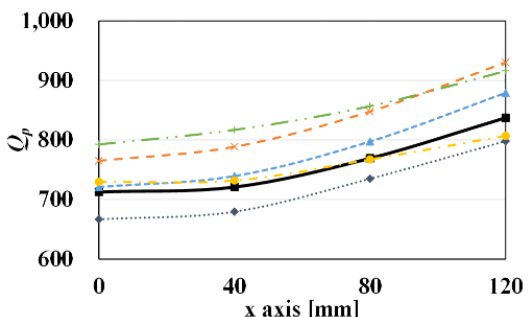

(g)

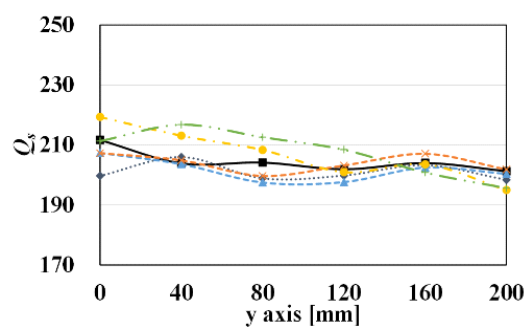

(j)

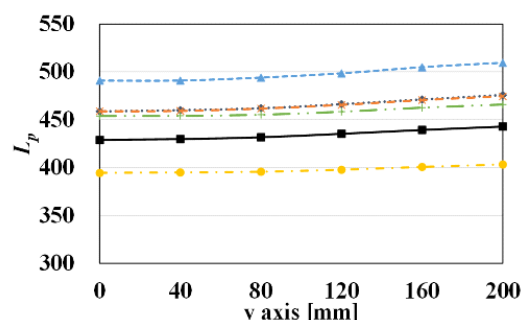

(b)

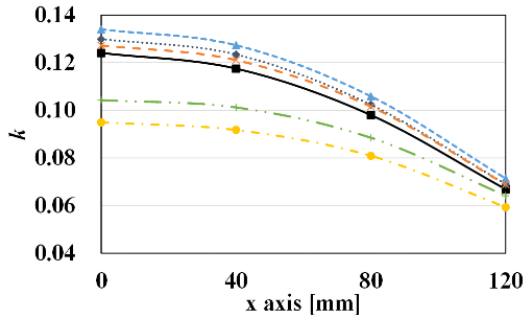

(e)

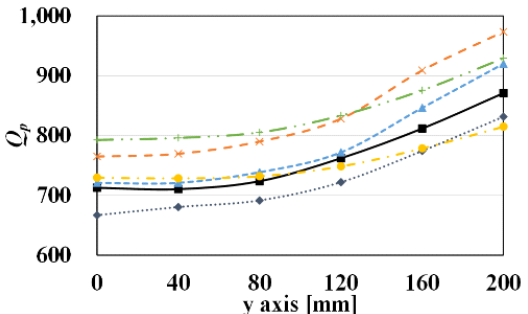

(h)

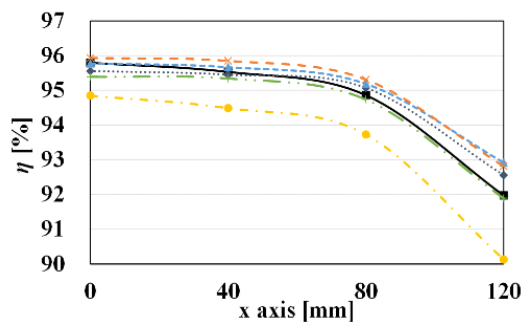

(k)

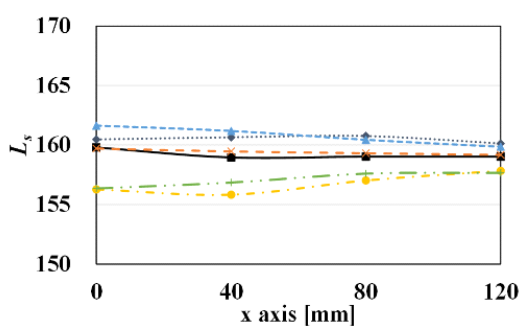

(c)

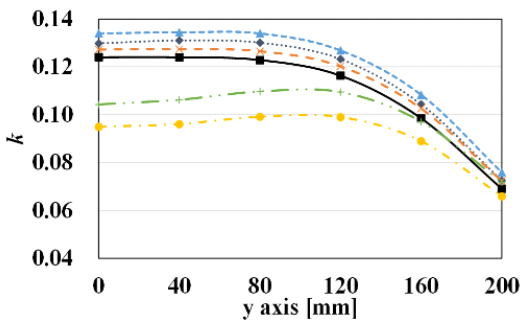

(f)

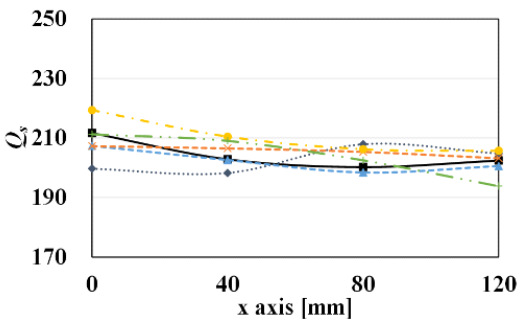

(i)

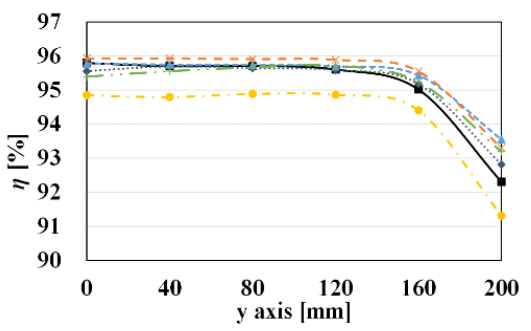

(1)

Fig. 6. Simulation results: (a), (b) primary pad inductance, (c), (d) secondary pad inductance, (e), (f) coupling coefficient, (g), (h) primary pad quality factor, (i), (j) secondary pad quality factor, and (k), (l) coil to coil efficiency

Table 1. Inductances and compensation capacitances of secondary pad at perfectly aligned position

\begin{tabular}{c|c|c|c|c|c|c}
\hline & case 1 & case 2 & case 3 & case 4 & case 5 & case 6 \\
\hline $\mathrm{L}_{\mathrm{s}}[\mu \mathrm{H}]$ & 159.80 & 160.46 & 161.62 & 159.71 & 156.27 & 156.36 \\
\hline $\mathrm{C}_{\mathrm{s}}[\mathrm{nF}]$ & 21.94 & 21.85 & 21.69 & 21.95 & 22.44 & 22.42 \\
\hline
\end{tabular}

illustrate the $Q_{s}$ of the six cases.

\subsection{Coil to coil efficiency}

The coil to coil transfer efficiency of the magnetic power pads can be achieved by applying current to the primary coil and resonating the pad inductance with compensation capacitance for both primary and secondary pads. The target output power for the simulation is set by $3.3 \mathrm{~kW}$. The secondary compensation capacitance, $C_{s}$, is designed at resonant frequency, and the primary compensation capacitance, $C_{p}$, is selected for tuning at resonant frequency. The designed compensation capacitance for each case is used regardless of the misalignment condition. Since the resonant network topology used for primary pad in this paper is LCL type, calculation of $C_{p}$ is not needed for simulation, instead, primary current, $I_{p}$, is designed as $16.83 \mathrm{~A}_{\mathrm{rms}}$ [10]. Table 1 represents the $L_{s}$ and $C_{\mathrm{s}}$, and in case of $L_{s}$, those are inductance values when the secondary pad is perfectly aligned, $x / y / z=0 / 0 / 100 \mathrm{~mm}$. Figs. $6(\mathrm{k})$ and (1) show the efficiency under $\mathrm{x}$ and $\mathrm{y}$ axis misalignment condition. Unlike the results for $k$ and $Q_{p}$, case 4 shows 


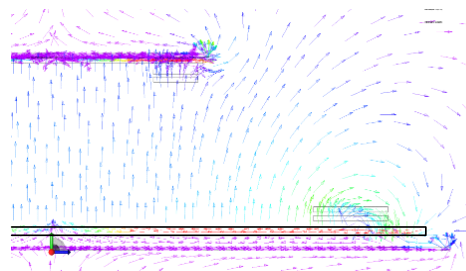

(a) case 1

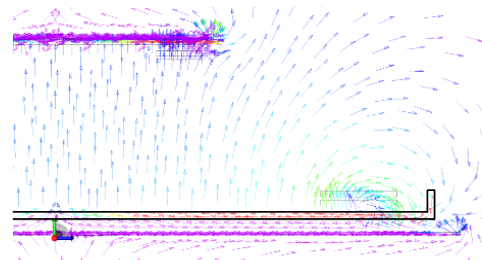

(d) case 4

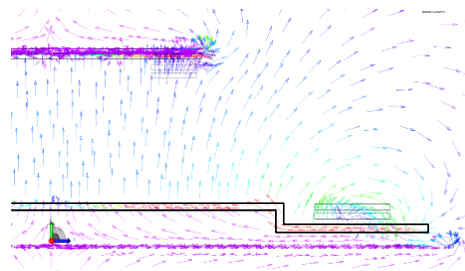

(b) case 2

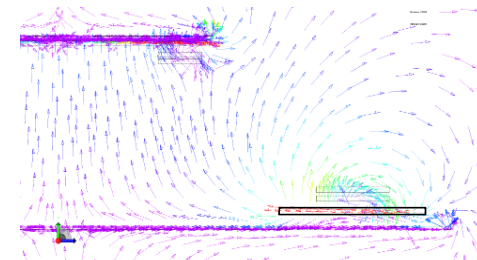

(e) case 5

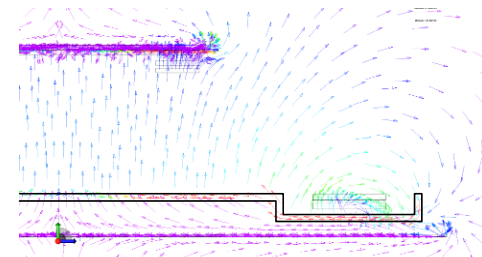

(c) case 3

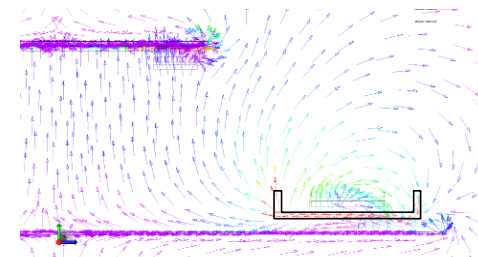

(f) case 6

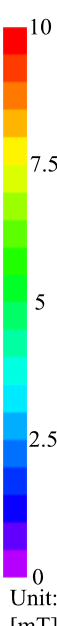

Unit:

Fig. 7. Magnetic flux density distribution according to the ferrite structure

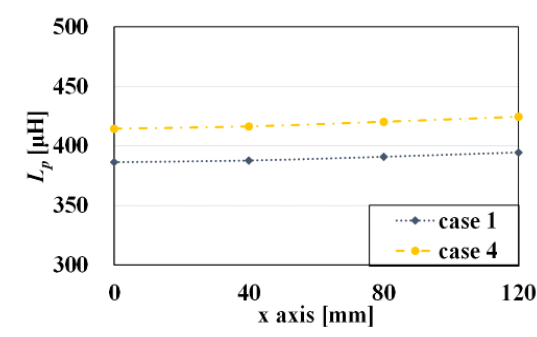

(a)

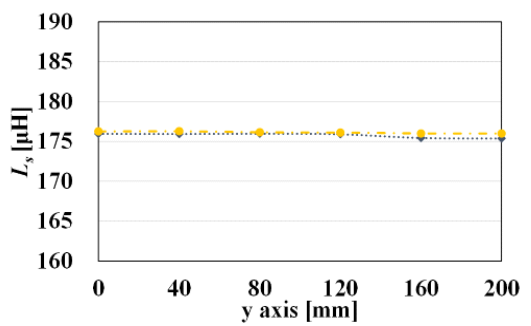

(d)

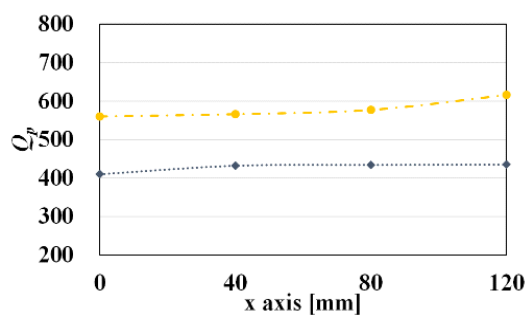

(g)

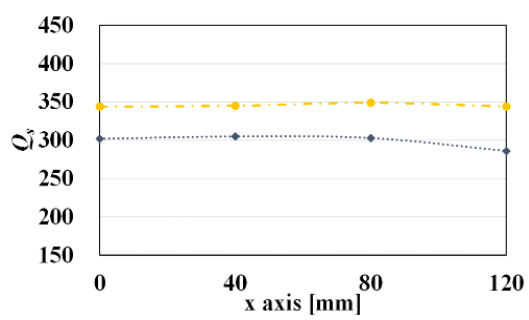

(i)

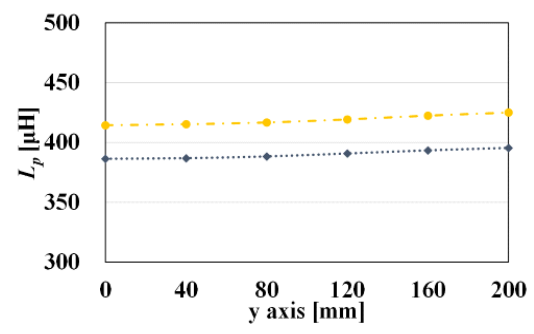

(b)

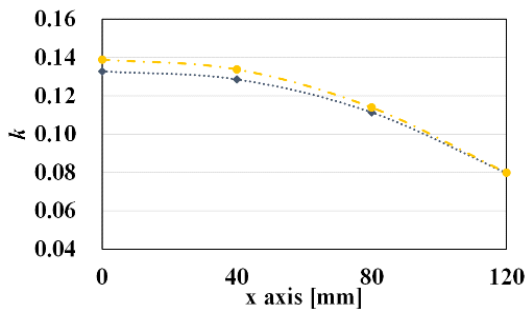

(e)

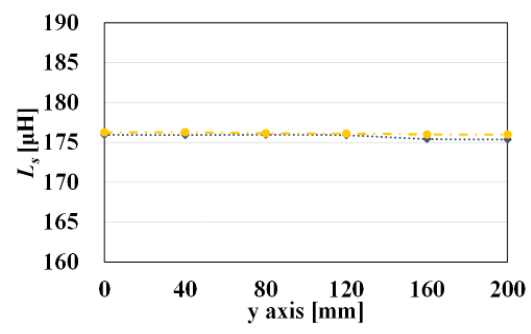

(c)

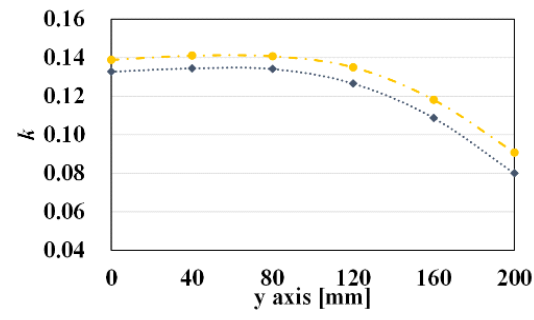

(f)

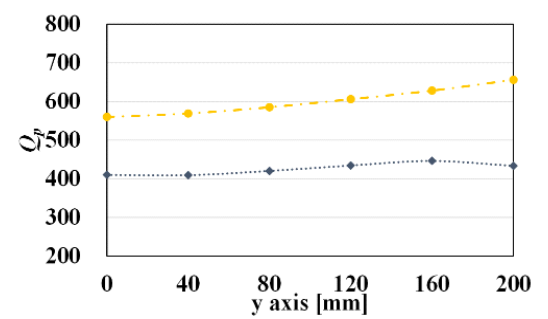

(h)

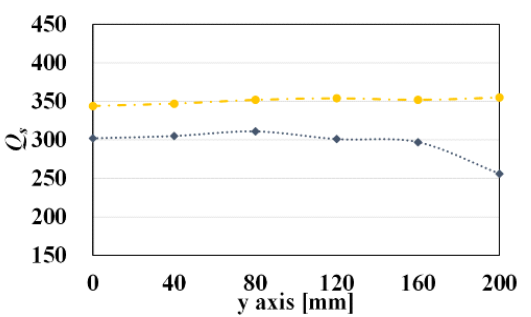

(j)

Fig. 8. Measured parameters: (a), (b) primary pad inductance; (c), (d) secondary pad inductance; (e), (f) coupling coefficient; (g), (h) primary pad quality factor, and (i), (j) secondary pad quality factor 
the highest efficiency over misalignment condition. The highest efficiency is $95.93 \%$ at perfectly aligned condition $(x / y / z=0 / 0 / 100 m m)$. The reason of this tendency is that the coupling coefficient of the case 4 is slightly lower or equal to the highest coupling coefficient, while $Q_{p}$ of the case 4 is fairly higher than other cases. This result proves that the efficiency of the magnetic power pad is not determined by only coupling or quality factor but both coupling and quality factor.

\subsection{Magnetic flux density}

In order to identify the effects of the ferrite structure variation on magnetic flux density, FEM simulation is performed. Figs. 7(a) to (f) illustrate the magnetic flux density when the $3.3 \mathrm{~kW}$ is delivered to the secondary power pad. The arrows represent the direction of the magnetic flux and their densities are expressed by color bar. As can be seen in the figures, the red arrows are more converged at the ferrite part, especially at either the protrusive part at the center or prolonged part at the outermost. Through the simulation results, it can be anticipated that the utilization of ferrite is beneficial for enhancing coupling between power pads.

\section{Magnetic Performance Verification}

Based on the FEM simulation results, two ferrite structure, which are case 1 and case 4 , are implemented and their magnetic parameters and performance are compared. Fig. 8 illustrates the parameter comparison of simulation and measured values. All parameters are measured using Hioki LCR HiTESTER 3522-50. The $L_{p}, L_{s}, k, Q_{p}$, and $Q_{s}$ of the case 4 are larger or superior than those of the case 1 . This results nearly coincides the tendency of the simulation results. However, in case of the quality factors, the magnitude of the manufactured power pads fairly lower than those of the simulation results. This is caused by unconcerned power loss factor in the simulation assumption.

\section{Conclusion}

In this paper, performance of magnetic power pads for IPT system with ferrite structure variation is analyzed. In order to evaluate the effects of the ferrite structure, six ferrite structures are suggested and their performance is compared in terms of primary and secondary selfinductance, coupling coefficient, primary and secondary quality factors, and coil to coil efficiency where secondary power pad is misaligned to either $\mathrm{x}$ or $\mathrm{y}$ axis. Through the simulation results, it is identified that protrusive ferrite at center of primary ferrite is helpful to increase of primary self-inductance and improvement of coupling (case 2 and
3 ). With regard to the primary quality factor, case 4 (flat plate ferrite with prolonged ferrite at outermost) and case 6 (enveloping coils with ferrite) are superior to other cases. The case 4 shows the highest coil to coil efficiency over misalignment condition and efficiency range is from $93.3 \%$ to $95.93 \%$. In order to investigate the effectiveness of the analysis using FEM simulation, two cases of the power pads, which use the ferrite structure of the case 1 and case 4 , are manufactured and their performance are compared. The measured results also correspond to those of the simulation results. Meanwhile, it is also proved that the primary ferrite structure does not have significant effect on the performance of either secondary self-inductance or quality factor.

\section{Acknowledgements}

This work was supported by "Human Resources Program in Energy Technology" of the Korea Institute of Energy Technology Evaluation and Planning(KETEP), granted financial resource from the Ministry of Trade, Industry \& Energy, Republic of Korea. (No. 20164030200980)

\section{References}

[1] H. Takanashi, Y. Sato, Y. Kaneko, S. Abe, and T. Yasuda, "A large air gap $3 \mathrm{~kW}$ wireless power transfer system for electric vehicles," in Proc. IEEE Energy Conversion Congress and Exposition (ECCE), Raleigh, NC, 2012, pp. 269-274.

[2] SAE International, "Wireless Power Transfer for Light-Duty Plug-In / Electric Vehicles and Alignment Methodology," [Online]. Available: http://standards.sae.org/wip/j2954/

[3] Mickel Budhia, Grant A. Covic, and John T. Boys, "Design and Optimization of Circular Magnetic Structures for Lumped Inductive Power Transfer Systems," IEEE Trans. Power Electron., vol. 26, no. 11, pp. 3096-3108, Nov. 2011.

[4] Venugopal Prasanth, Pavol Bauer, and J.A. Ferreira, "A Sectional Matrix Method for IPT Coil Shape Optimization," in Proc. $9^{\text {th }}$ International Conference on Power Electronics - ECCE Asia (ICPE-ECCE Asia 2015), Seoul, 2015, pp. 1684-1691.

[5] Shuo Wang and David G. Dorrell, "Loss Analysis of Circular Wireless EV Charging Coupler," IEEE Trans. Mag., vol. 50, no. 11, Nov. 2014.

[6] Siqi Li and Chunting Chris Mi, "Wireless Power Transfer for Electric Vehicle Applications," IEEE J. Emerg. Select. Topics Power Electron., vol. 3, no. 1, pp. 4-17, Mar. 2015.

[7] J. Mühlethaler, "Modeling and multi-objective optimization of inductive power components," Ph.D. dissertation, Dept. Elect. ENg., ETHZ, Zürich, The 
Switzerland, 2012.

[8] Mickel Budhia, John T. Boys, Grant A. Covic, Chang-Yu Huang, "Development of a Single-Sided Flux Magnetic Coupler for Electric Vehicle IPT Charging Systems," IEEE Trans. Ind. Electron., vol. 60, no. 1, Jan. 2013.

[9] Fey Yang Lin, Grant A. Covic, John T. Boys, "Evaluation of Magnetic Pad Sizes and Topologies for Electric Vehicle Charging," IEEE Trans. Power Electron., vol. 30, no. 11, Nov. 2015.

[10] Mangesh Borage, Sunil Tiwari, and Swarna Kotaiah, "Analysis and Design of an LCL-T Resonant Converter as a Constant-Current Power Supply," IEEE Trans. Ind. Electron., vol. 52, no. 6, Dec. 2005.

[11] Minkook Kim, Jongeun Byeon, Jae-Woo Lee, and Byoung Kuk lee, "Performance Analysis of Magnetic Power Pads for Inductive Power Transfer Systems with Ferrite Structure Variation," in Proc. IEEE Energy Conversion Congress and Exposition (ECCE), Milwaukee, WI, 2016, pp. 1-6.

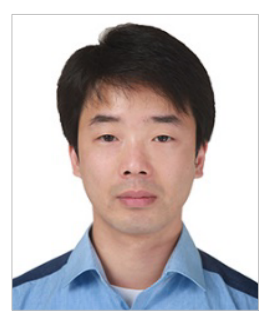

Minkook Kim He received the B.S degree in electrical engineering from Sungkyunkwan University, Suwon, Korea, in 2012, where he is currently working toward the combined M.S. and Ph.D. degree in electrical engineeering. His research interest includes wireless power transfer system for

electric vehicles.

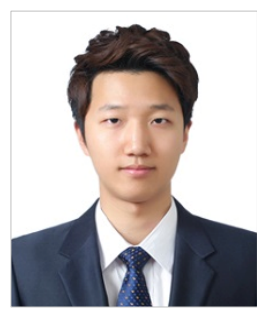

Jongeun Byun He received the B.S degrees in electrical engineering from Sungkyunkwan University, Suwon, Korea, in 2015, where he is currently working toward the combined M.S and Ph.D. degree in electrical and computer engineering. His research interests include wireless power transfer and energy storage system.

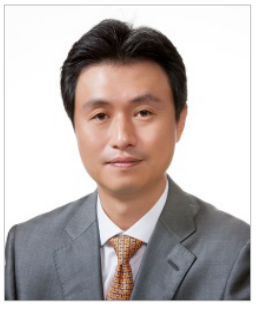

Byoung Kuk Lee He received the B.S. and the M.S. degrees from Hanyang University, Seoul, Korea, in 1994 and 1996, respectively and the Ph.D. degree from Texas A\&M University, College Station, TX, USA, in 2001, all in electrical engineering. From 2003 to 2005, he was a Senior Researcher with Power

Electronics Group, Korea Electrotechnology Research Institute, Changwon, Korea. From 2006, he is with the College of Information and Communication Engineering, Sungkyunkwan University, Suwon, Korea. His research interests include on-board charger and wireless power transfer for electric vehicles, energy storage systems, hybrid renewable energy systems, dc distribution systems for home appliances, power conditioning systems for fuel cells and photovoltaic, modeling and simulation, and power electronics. Prof. Lee received the Outstanding Scientists of the 21st Century from IBC and listed on 2008 Ed. of Who's Who in America and 2009 Ed. of Who's Who in the World. He is an Associate Editor in the IEEE TRANSACTIONS ON INDUSTRIAL ELECTRONICS and Guest Associate Editor in the IEEE TRANSACTIONS ON POWER ELECTRONICS. He was the Presenter for Professional Education Seminar with the topic of "OnBoard Charger Technology for EVs and PHEVs" at the IEEE Applied Power Electronics Conference in 2014 and was the General Chair for the IEEE Vehicular Power and Propulsion Conference in 2012. 